\title{
Effect of Hydrogen Additive on the Evolution of Beam Divergence in Copper Bromide Lasers
}

\author{
Peter Graham Foster, Peter Eric Davis, Donald McCoy, and Jesper Munch
}

\begin{abstract}
We present a systematic study of the effect of hydrogen additive on the beam quality of copper bromide lasers. It is a well-established fact that the addition of small amounts of hydrogen to the neon buffer gas of this class of laser will dramatically increase their average output power and efficiency. Our results show that the partial pressure of hydrogen used to maximize the total output power is not the same as that which optimizes the power density in the drilling plane of a copper bromide laser based micro-machining system.
\end{abstract}

Index Terms-Laser, laser material processing..

\section{INTRODUCTION}

C OPPER vapor lasers (CVLs) are currently the highest average power visible wavelength lasers available, emitting several hundred watts of average power on transitions in the green $(510.6 \mathrm{~nm})$ and yellow $(578.2 \mathrm{~nm})$ at pulse repetition frequencies of tens of kilohertz. Initial development of the CVL was based on the need for a high average power pump source for the tunable dye lasers used in atomic vapor laser isotope separation (AVLIS) [1]. The copper bromide (CuBr) laser was one of a class of copper halide lasers developed in response to the high operational temperatures of the elemental CVL [2]. The efficiency and beam quality of elemental CVLs have recently been improved by adding hydrogen halides to the flowing neon buffer gas [3], [4].

The $\mathrm{CuBr}$ laser has not received the research attention of the CVL due to the difficulties of producing long lifetime sealed systems. These problems have now been largely overcome. The low operating temperature of the $\mathrm{CuBr}$ laser, around $500{ }^{\circ} \mathrm{C}$ [5], allows use of an all-fused silica laser tube instead of high-temperature ceramics, and the lower outgassing rates at these temperatures obviates the need for a flowing gas system. The low temperature of the $\mathrm{CuBr}$ laser also results in rapid warm-up and cool-down times compared with the CVL. Copper hydrogen-halide flowing gas systems also have improved power and beam quality and operate at lower temperatures, but the cost and maintenance of pumping corrosive gases and their disposal detract significantly from the advantages of these systems.

Manuscript received April 9, 2001; revised August 20, 2001. This was supported in part by an Australian Commonwealth Government SPIRT Grant.

P. G. Foster is with the Lasers and Non-Linear Optics Group, Department of Physics and Mathematical Physics, University of Adelaide, Adelaide Australia, and also with Fiberbyte, Adelaide 5000, Australia (e-mail: pfoster@physics.adelaide.edu.au).

P. E. Davis is with the Norseld Laser Systems, Adelaide, Australia.

D. McCoy and J. Munch are with the Lasers and Non-Linear Optics Group, Department of Physics and Mathematical Physics, University of Adelaide, Adelaide, Australia.

Publisher Item Identifier S 0018-9197(01)10247-2.
Medical applications have provided the proving ground for sealed $\mathrm{CuBr}$ laser technology, being the preferred laser due to the smaller size, fast warm-up time, and freedom from pumps and flowing gas. The yellow line of the $\mathrm{CuBr}$ laser emission occurs near the peak of the oxy-hemaglobin absorption in the low melanin absorption regime providing optimum treatment of port wine stains and telangiactasia [6] while the green line is well suited for treatment of pigmented lesions and cutting procedures such as palatoplasty.

In recent years, interest has emerged in the application of copper lasers to precision micro-machining [7] and nonlinear frequency conversion to the ultraviolet (UV) [8]. For best results, these applications require a laser of excellent beam quality. Considerable effort has gone into characterization of elemental CVLs [9]-[11], and more recently the copper hybrid (Cu-HyBrID) [12] and kinetically enhanced (KE)-CVLs [13], for these tasks, but, until now, little similar effort has been applied to the $\mathrm{CuBr}$ laser.

$\mathrm{Cu}-\mathrm{HyBrID}$ and KE-CVL lasers show significantly improved output power and high-beam-quality extraction over their elemental counterparts. Reports in the literature describe high-beam-quality output in the 50-100-W range. A KE-CVL master oscillator-power amplifier (MOPA) delivering in excess of $200 \mathrm{~W}$ at 2.5 times the diffraction limit [14] has also been reported. These systems are based on high-temperature, largescale systems. The $\mathrm{CuBr}$ laser oscillator presented here is compact and efficient, producing around $7 \mathrm{~W}$ of high-beam-quality emission.

This study was motivated by an observation that laser cutting of metals using our $\mathrm{CuBr}$ MOPA arrangement was far more efficient and produced a cleaner cut when using an old, low-power master oscillator laser tube than when a new high-power tube replaced it. In this paper, we describe recent work on high-beamquality $\mathrm{CuBr}$ lasers. In particular, we examine the effect of hydrogen additive on the laser's ability to precisely deliver energy to the machining target material.

\section{THE CUBR LASER}

\section{A. The Laser System}

The oscillator and single-amplifier $\mathrm{CuBr}$ laser system used here (Norseld Laser Systems) can be operated at up to $40 \mathrm{~W}$ of average power at a $16-\mathrm{kHz}$ pulse repetition frequency (prf). This is a limit only of the high-voltage power supply used in this particular system.

The laser discharge tube is of $45-\mathrm{mm}$ bore and 500-mm active length, with the discharge being limited by $20-\mathrm{mm}$ quartz apertures placed along the length of the tube. The total length of the 


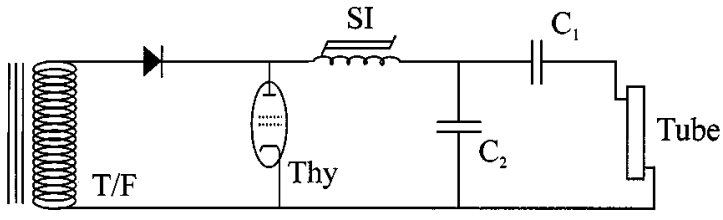

Fig. 1. High-voltage excitation circuit. T/F: high-voltage transformer. Thy: thyratron. SI: saturable inductor. $C_{1}, C_{2}$ : capacitors.

laser tube is $1 \mathrm{~m}$, including cooled sections at either end that are used as bromine traps to prevent an increase in bromine partial pressure which can seriously degrade laser system performance over time.

The copper bromide lasant donor is located in three independently heated side-arm reservoirs which enable a controlled, uniform distribution of $\mathrm{CuBr}$ vapor along the length of the active region. The electrodes are made from copper and placed in side arms with tungsten "bright seal" electrical feedthroughs.

The system features an actively stabilized laser timing control system based on a 2-GHz oscillator. A small air-cooled thyratron is used to switch approximately $1 \mathrm{~kW}$ of average power in each tube at a repetition rate of $16 \mathrm{kHz}$. Saturable inductors are used in the excitation circuitry to minimize the power load on the thyratron to extend its lifetime, as shown in Fig. 1. The high-voltage circuit is fed from a small command charge power supply which eliminates the crowbar problems common with resonantly charged power supplies.

\section{B. Divergence Evolution in Copper Atom Lasers}

The $\mathrm{CuBr}$ laser operates on transitions from resonance to metastable levels in neutral copper atoms produced by dissociation of $\mathrm{CuBr}$ vapor in an electrical discharge. Like other members of the metal vapor laser family, the free copper atoms are excited by electron impact using electrical discharges supported in low-pressure neon. The lower level being metastable means that the transitions are self terminating. The extremely high gain $\left(\sim 10^{5}\right)$ is thus short-lived. It has been established by Coutts and Brown [15] that all output from the CVL is essentially propagation and amplification of an initial radiation seed and that beam divergence improves in discrete steps corresponding to successive round trips of the seed when the laser employs the typical high magnification unstable resonator.

In the present experiments, only the oscillator was used. It used a high-magnification ( $M=62$ ) positive branch confocal unstable resonator. The rear dielectric mirror $(R=2115 \mathrm{~mm})$ was highly reflecting at both laser wavelengths and the output coupler, a 1-mm-diameter spot reflector, was similarly coated. The outcoupling mirror $(R=34.15 \mathrm{~mm}$ ) was constructed by polishing a bundle of 1-mm-diameter silica fibers which were coated for high reflectivity. One of the fibers was then supported on a molybdenum foil spider in the beam to act as the outputcoupler.

\section{EXPERIMENT}

\section{A. Gas-Handling System}

The gas-handling system used a turbomolecular pump, backed by an oil-filled rotary vane pump, to produce pressures in the range of $10^{-8}$ torr. In order to ensure the cleanliness of the laser tubes, rough pumping was performed by a molecular drag pump which was backed by an oil-free diaphragm pump. Gas pressures were measured in the 0.01-100-torr range by capacitance manometer gauges and lower pressures were measured with cold cathode gauges. In addition to this, a mass spectrometer was used for trace gas identification.

\section{B. Laser Tube Setup}

Reliable hydrogen pressure readings were ensured by repeatedly purging the tube of hydrogen before each data run. The laser was operated at normal operating temperatures using a charge of pure neon only and then quickly pumped to high vacuum using the turbo-molecular pump.

The capacitance manometers, used for gas mixture measurement, were zeroed before each gas charge of the tube. The zero drift of these devices was only \pm 0.01 torr during the entire experiment. Total cold laser tube pressure used in these experiments was always 26 torr, regardless of the amount of added hydrogen.

\section{Beam Characterization}

Time-resolved studies of the evolution of the output of the laser oscillator have been conducted using power-in-the-bucket measurements [10]. The measurement system comprised a long effective focal length $(\sim 25 \mathrm{~m})$ telescope using achromatic lenses that produced a moderately sized far-field spot for measurement, as shown in Fig. 2. The amount of energy that passed through an aperture placed in the far-field was measured as a function of time using an integrating sphere containing a fast (3-ns risetime) photodiode and 300-MHz (Tektronix 2440) oscilloscope and also with an average power meter (Gentec PS-25). The effective focal length of the diagnostic system telescope was calibrated by measuring the lateral displacement of the far-field spot when introducing a known angular displacement of the beam.

The laser beam quality was then determined by numerical integration of the temporal profiles of the optical pulse when different size apertures were used in the far-field. The variation in beam quality throughout the pulse could also be analyzed using the same integration technique but with a smaller integration time.

Open air turbulence was a significant problem with this diagnostic system because the focal plane of the telescope was several meters from the telescope. These effects were reduced by enclosing the beam path in a length of PVC water pipe.

The voltage across the laser tube was measured with Tektronix P6015A high-voltage probes. Current flowing in the tube was measured with a Pearson (model 110) pulse current transformer. This was checked by current measurements using Rogowski coils of varying speed.

\section{RESULTS}

\section{A. Total Output Power}

Some reports [5] indicate that the average output power of the $\mathrm{CuBr}$ laser almost doubles when $2 \%$ hydrogen is added to the 


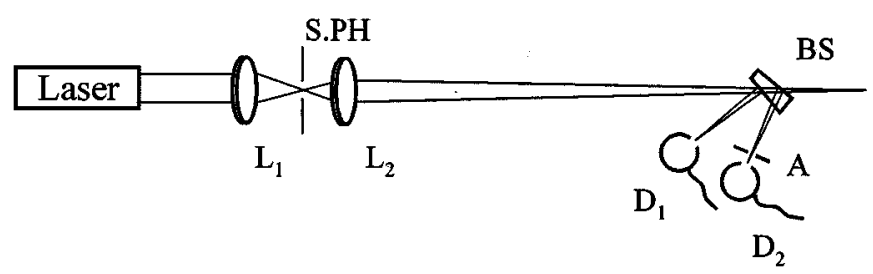

Fig. 2. Diagnostic system used for far-field beam divergence characterization of the laser system. $L_{1}, L_{2}$ : telescope achromatic lenses. BS: wedged beamsplitter. A: aperture. $D_{1}, D_{2}$ : detectors. S.PH: soft pinhole for ASE rejection. ( $L_{1}: 500 \mathrm{~mm} \mathrm{EFL;} L_{2}: 100 \mathrm{~mm}$ EFL; separation of telescope lenses $\sim 600 \mathrm{~mm}$ ).

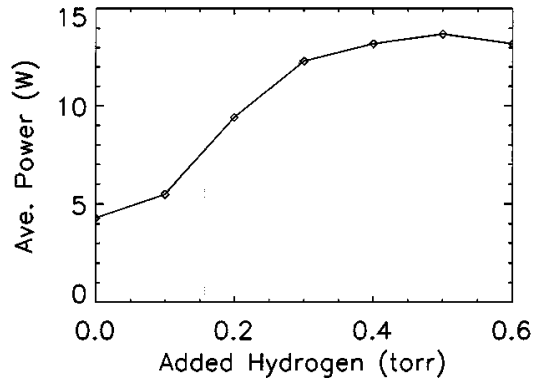

Fig. 3. Average laser power as a function of the partial pressure of hydrogen.

laser tube. We have found that other impurities, such as water vapor and oxides, in the laser tube perform a similar function (but not to the same extent) to that of adding hydrogen. "Dirty" laser tubes have an output beam that is predominantly annular, but as they are further cleansed in the absence of hydrogen, the output power drops and the beam becomes more annular with a deeper central minimum. We have found that a clean tube operating in the absence of hydrogen with an output of around $4 \mathrm{~W}$ can be increased to almost $14 \mathrm{~W}$ with the addition of the optimal amount of hydrogen. This is an improvement of well over three times. Fig. 3 shows that further addition of hydrogen reduced the total optical output from the laser.

\section{B. Amplified Spontaneous Emission}

A set of measurements were made of the laser pulse shape when operated with different partial pressures of hydrogen. Fig. 4 shows the pulse shape of these emissions normalized to the peak height of the non-amplified spontaneous emission (ASE) (or signal) component of the pulse. This indicates the extent to which the initial ASE spike contributes to the total output.

The ASE content in the laser output was determined by comparison of the average laser power with and without a "soft pinhole" of $2 \mathrm{~mm}$ in diameter (corresponding to about 50 times the diffraction limit) placed at the focal plane of the first lens $(f=0.5 \mathrm{~m})$ (see Fig. 2). This rejected all light having a divergence exceeding $4 \mathrm{mrad}$ and has undergone at most one round trip of the resonator.

When the laser was operated without hydrogen, the gain was annular and half of the emission was in the form of ASE at 510 $\mathrm{nm}$. At hydrogen pressures that maximized the average power of the laser (0.5 torr), the initial ASE spike contained just over $20 \%$ of the pulse energy. Further increases in hydrogen pressure decreased the total power and increased the percentage of ASE in the beam. The contribution of the initial ASE spike was min-

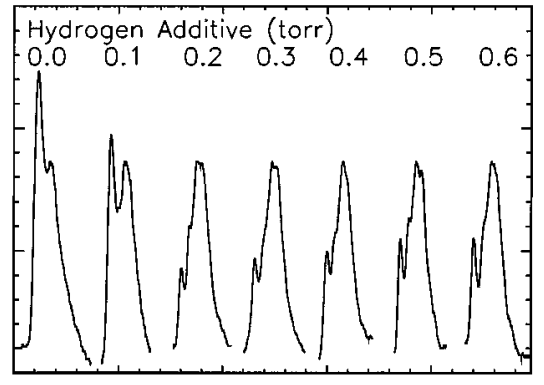

Fig. 4. Measurements of the laser pulse shape with different partial pressures of hydrogen added to the tube. These waveforms have been normalized to the peak height of the non-ASE component of the emission to illustrate the contribution made by the initial ASE spike to the output.

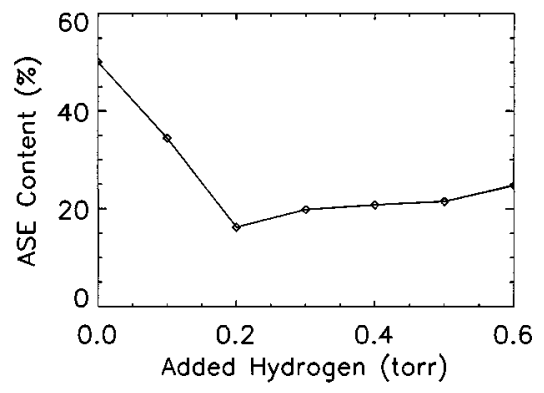

Fig. 5. The proportion of ASE in the laser emission as a function of the partial pressure of hydrogen.

imized to approximately $9 \%$ at a hydrogen pressure of 0.2 torr $(0.8 \%)$ as can be seen in Fig. 5.

ASE throughout the pulse contributed about $7 \%$ to the total emission when the initial ASE spike was ignored. This contribution was almost the same for all hydrogen pressures above 0.2 torr.

The total ASE content, including the initial ASE spike, was found to be minimized (about 16\%) when operating the laser with 0.2 torr of added hydrogen. This can be attributed to the slower rise in gain and gain saturation effects as will be discussed in detail in Section IV-D.

The temporal shape of the spontaneous emission from the tube without a resonator was also measured. The output from the tube was sampled using Fresnel reflection from an uncoated BK7 wedge. The reflection was then focussed through a 3-mmdiameter aperture with an anti-reflection coated $f=100 \mathrm{~mm}$ achromat and measured using the integrating sphere. Great care was taken to prevent unwanted reflections being fed back into the tube, including a low back-reflection beam dump located 


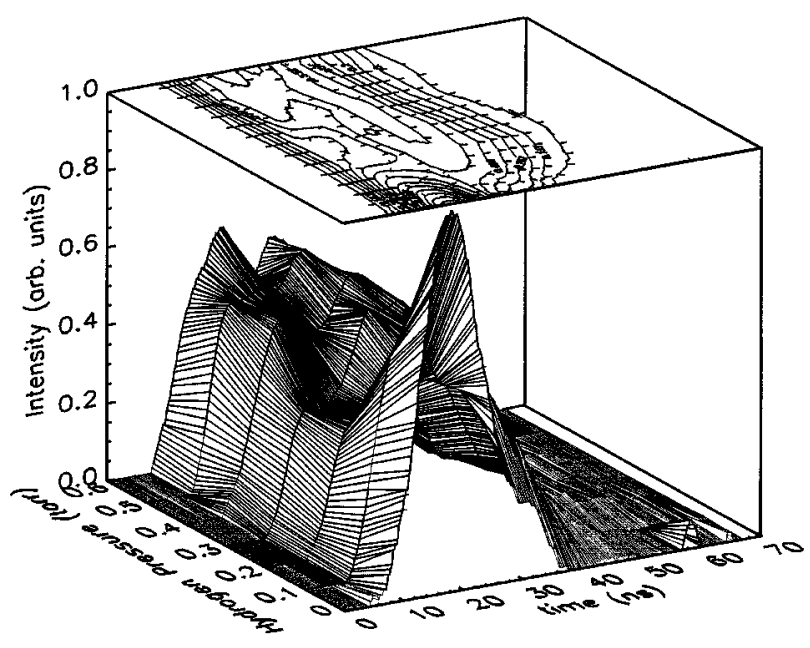

Fig. 6. Mirrorless ASE from the tube as a function of partial hydrogen pressure.

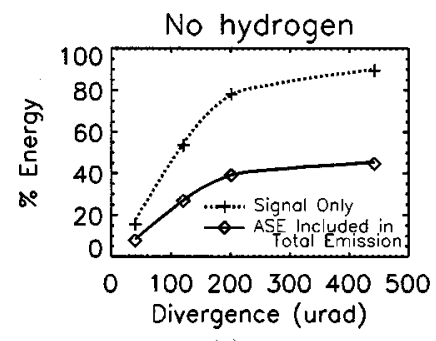

(a)

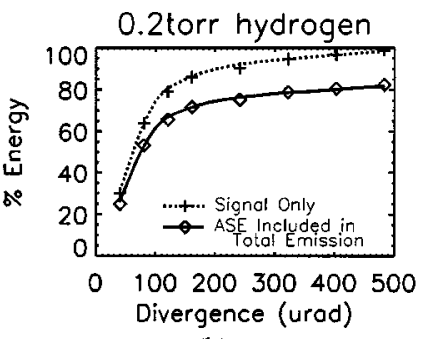

(b)
Fig. 7. The effect of including the contribution made by ASE in the beam divergence measurements. (a) Neon-only buffer gas. (b) With 0.2 torr of added hydrogen.

several meters behind the laser tube. Using this arrangement, the temporal profile of the mirrorless ASE was recorded for different hydrogen pressures. The emission at each pressure was normalized to have the same area and compiled on one graph (Fig. 6).

The important point to note is the relative strength of the emission throughout the pulse as we change hydrogen pressure. With a neon-only buffer gas, the emission occurs in essentially one large pulse. As some hydrogen is added, the pulse length increases and we begin to see the emergence of two distinct peaks. At 0.2 torr, we find that the first peak is minimized and the following peak is about twice the intensity. The pulse appears widest at a pressure of 0.5 torr which corresponds to the maximum average power achievable from the system but the two peaks are now about the same intensity. When the hydrogen pressure is further increased, the first peak again dominates.

Changes in ASE dynamics with different hydrogen gas admixtures are due primarily to the changed plasma conditions prior to the excitation pulse. Dissociative attachment of free electrons by the $\mathrm{HBr}$ molecule is responsible for reducing the pre-pulse electron density [17], [19], [20]. This leads to a significant reduction in the plasma skin effect which radically modifies the radial beam profile (as discussed later in Fig. 9).

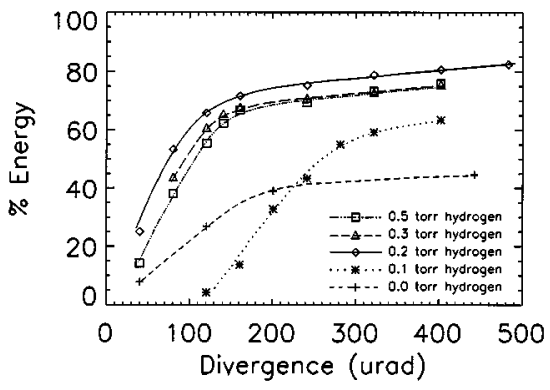

Fig. 8. Far-field divergence measurements as a function of the partial pressure of hydrogen in the tube.
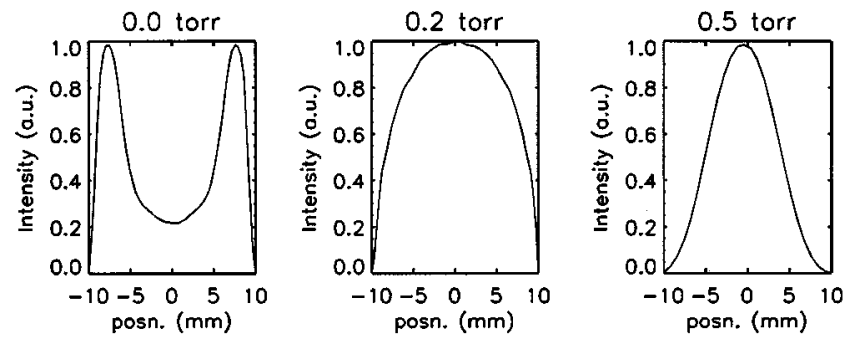

Fig. 9. Near-field energy distribution of the laser emission as a function of partial pressure of hydrogen in the system.

\section{Power-in-the-Bucket}

Most reports simply describe the evolution of the low divergence components of the output of such lasers. The ASE component is often ignored since it will be spatially filtered from the output beam prior to use in machining systems or second-harmonic generation. This gives an unrealistic impression of the efficiency of generating low divergence emission.

When one includes the amount of ASE in the output, the proportion of energy at low divergence drops considerably. When there is no added hydrogen, half of the emission occurs as ASE. The minimum ASE is reached at 0.2 torr of hydrogen and a greater proportion of the power is delivered into the low divergence components as discussed in Section IV-B. Fig. 7(a) and (b) show the effect of including the ASE component in power-in-the-bucket results.

The far-field spot size, corresponding to the turnover point of each curve (Fig. 8), is slightly different for hydrogen pressures above 0.2 torr. This was also noted by Sabotinov [21]. When we added 0.5 torr of hydrogen to the tube, the far-field divergence angle (at the $1 / e^{2}$ point) became $45 \%$ larger than when operating with 0.2 torr. The energy can be deposited into the smallest possible focal spot when operating with 0.2 torr of hydrogen.

We have determined that this is due to modification of the near-field energy distribution that occurs with changes in hydrogen pressure. The profile is annular with no hydrogen, then becomes what can loosely be described as a top-hat distribution at 0.2 torr and then predominantly Gaussian in nature at higher pressures (see Fig. 9).

We have calculated numerical Fourier transforms of the various near-field intensity distributions corresponding to different 


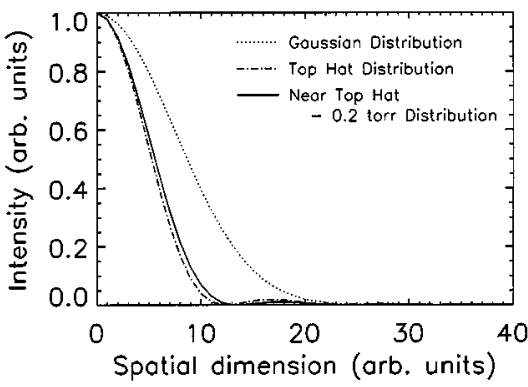

Fig. 10. Numerical simulation of the far-field energy distribution corresponding to diffraction-limited propagation of various near-field intensity distributions.

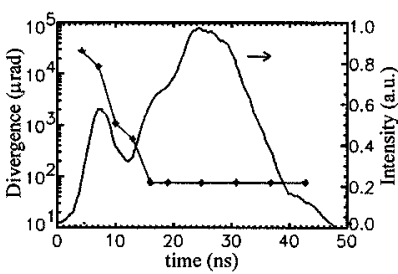

(a)

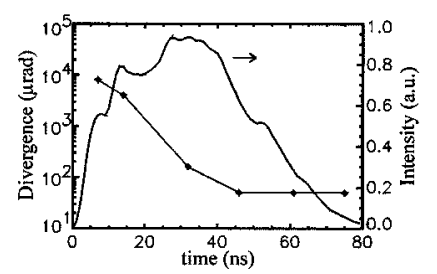

(b)
Fig. 11. Comparison of the emission from our $\mathrm{CuBr}$ laser and an industrial scale elemental CVL. Divergence data is calculated from theory. (a) $\mathrm{CuBr}$ laser. (b) CVL.

hydrogen partial pressures used. The theoretical $1 / e^{2}$ far-field spot diameter of the Gaussian (0.5 torr) distribution is $48 \%$ larger than that of the near top-hat (0.2 torr) distribution (see Fig. 10). Our measured differences in the far-field divergence angle are in good agreement with propagation of the real nearfield distributions into the far-field. The amplitude shape and aperture size of the near-field profile determines the ability to focus in a given optical system. A near-field Gaussian intensity distribution by itself does not ensure maximum energy in a tightly focused spot. What is important is the combination of amplitude apodization and phase control (beam quality) to maximize power on the target.

Section IV-B described the observation that the ASE content of the laser pulse was also minimized when operating with 0.2 torr of hydrogen. This is a fortunate coincidence. The optimum operating conditions for low divergence emission are the same as those required for maximum efficiency in delivering the available output power into that low divergence component.

When the laser was operated with 0.2 torr of added hydrogen, the maximum achievable output power was $9.8 \mathrm{~W}$ in a plane-plane cavity arrangement, which dropped only slightly to $9.5 \mathrm{~W}$ when operated with the $M=26$ unstable cavity. Filtering the ASE component from the emission left $6.7 \mathrm{~W}$ of emission at 2 times the diffraction limit.

\section{Time-Resolved Divergence Measurements}

In this section, we discuss the evolution of beam divergence in the $\mathrm{CuBr}$ laser. The emission from the oscillator has been observed to show temporal modulation of the optical output that is typical for copper lasers [Fig. 11(a)].

The optical pulse from the $\mathrm{CuBr}$ laser, however, has a small initial ASE spike, followed by a period of reduced gain before the intensity rises again toward the peak value. This means that

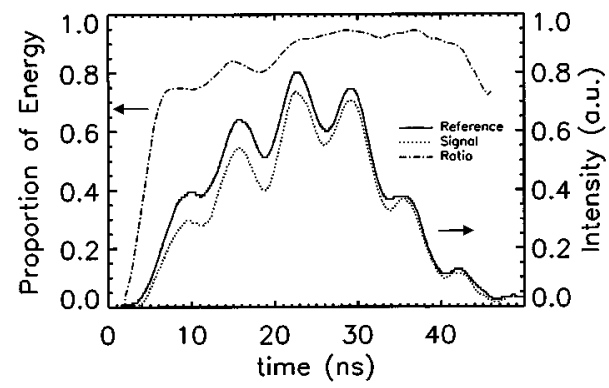

Fig. 12. Evolution of the beam divergence in our $\mathrm{CuBr}$ laser with the presence of the ASE seed pulse and subsequent gain saturation.

minimal energy is expended during formation of the low divergence component.

In addition, these lasers operate at a much lower wall temperature than their elemental counterparts. The resulting lower input power requirements lead to smaller radially dependent reductions in the neutral lasing species (for example, ionization by electron collisions). It is therefore possible to build a small system and still maintain uniform pressure of the lasing species across the gain volume. After three round trips of the short resonator, about $18 \mathrm{~ns}$ into the pulse, we are near the diffraction limit of $62 \mu \mathrm{rad}$, and this occurs before the peak in output intensity. In particular, there appears to be a substantial reduction of the second round-trip component ( $225 \mu \mathrm{rad}$ for the $M=62$ cavity).

For comparison, we studied an elemental CVL with $2 \% \mathrm{H}_{2}$ at the University of Bayreuth, Germany. The laser was located in the laboratories of the ATZ-EVUS, Vilseck, Germany. The output of the CVL oscillator can be seen in Fig. 11(b). The peak intensity occurs during the second round-trip component of the emission, the divergence of which is still many times the diffraction limit.

Temporal analysis of the divergence evolution of the $\mathrm{CuBr}$ pulse was performed. For this purpose we chose to operate the laser with our "optimum" hydrogen level of 0.2 torr. Initially, the beam had large divergence, as expected, which rapidly collapsed toward the diffraction limit. Fig. 12 shows the (non-ASE) pulse profile both before (reference beam) and after (signal beam) passing through a $2-\mathrm{mm}$ aperture in the far-field ( $80 \mu \mathrm{rad})$. Numerical integration of these profiles shows the instantaneous beam divergence.

We have produced 8- $\mu$ m-diameter holes in aluminum foils of $25-\mu \mathrm{m}$ thickness with single $\mathrm{CuBr}$ laser pulses. This is comparable to the $6.2-\mu \mathrm{m}$ diffraction-limited focal spot size (using a 100-mm effective focal length (EFL) achromatic focusing element at $f / 5$ [see Fig. 13(a). For comparison, Fig. 13(b) (courtesy of Dr. Mayerhofer) shows a single pulse hole produced by the CVL using a $120-\mathrm{mm}$ EFL achromat at $f / 4.8$. This hole is of the order of $30 \mu \mathrm{m}$ in diameter; however, the important point is the comparison of the heat-affected area around the two drill holes of Fig. 13. The area around the CVL drilled hole shows material removal (surface profile measurements) which is consistent with the diameter of the focused second round-trip component of the emission from this laser. In contrast, there is no observable heat damage around the hole drilled with the $\mathrm{CuBr}$ laser which supports the assertion that there is minimal energy expended before the emission reaches the diffraction limit. 


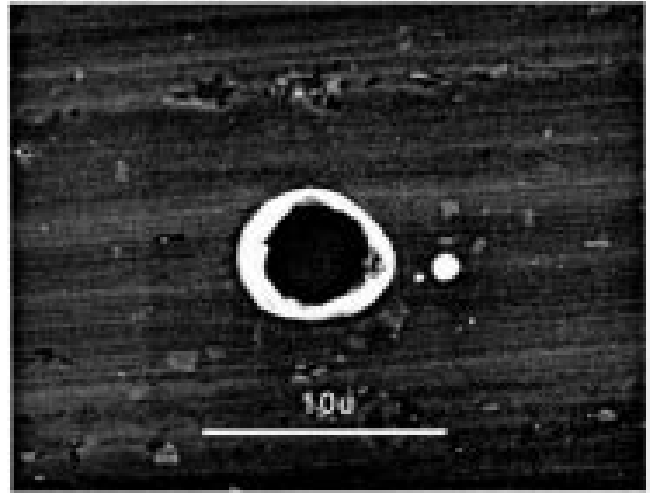

(a)

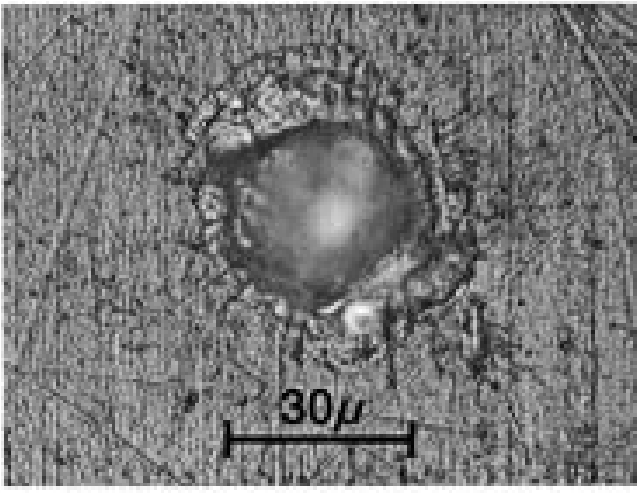

(b)

Fig. 13. Single-pulse drill holes in aluminum. (a) CuBr laser. (b) CVL.

\section{CONCLUSION}

At "optimum" hydrogen partial pressure ( 0.2 torr) in the 26-torr neon buffer gas, the $\mathrm{CuBr}$ laser produces a low percentage of ASE in the output laser pulse. Fortunately, the same hydrogen partial pressure results in a laser near-field distribution that maximizes the energy deposited into the smallest spot on the target material. Under optimum conditions, this system produces $6.7 \mathrm{~W}$ power having a divergence of twice the diffraction limit. The resulting system is capable of efficient micro-machining with little collateral damage around the drill site.

\section{ACKNOWLEDGMENT}

The authors would like to thank Dr. Mayerhofer and the late Prof. Bergmann of the Department of Metallic Materials, University of Bayreuth, Germany, and Mr. Hartmann of ATZ-EVUS, Vilseck, Germany, for access to the CVL used in this work.

\section{REFERENCES}

[1] B. Warner, "Atomic vapour laser isotope separation," Proc. SPIE, vol. 737, pp. 2-6, 1998.

[2] C. S. Liu et al., "Copper superradiant emission from pulsed discharges in copper iodide vapor," Appl. Phys. Lett., vol. 23, pp. 92-93, 1973.

[3] E. S. Livingstone et al., "Characteristics of a copper bromide laser with flowing $\mathrm{Ne}-\mathrm{HBr}$ buffer gas," Opt. Quantum Electron., vol. 24, pp. 73-82, 1992.

[4] M. J. Withford et al., "Investigation of the effects of hydrogen and deuterium on copper vapor laser performance," Opt. Commun., vol. 110, pp. 699-707, 1994.

[5] Astajov et al., "Parametric study of the CuBr laser with hydrogen additives," IEEE J. Quantum Electron., vol. 24, pp. 1927-1927, 1988.

[6] S. E. McCoy, "An evaluation of the copper-bromide laser for treating telangiactasia," Lasers in Surg. Med., vol. 21, pp. 329-337, 1997.

[7] C. Körner et al., "Physical and material aspects in using laser pulses of nanosecond duration for ablation," App. Phys. A, vol. 63, pp. 123-126, 1996.

[8] D. W. Coutts, "Time resolved beam divergence from a copper vapor laser with unstable resonator," IEEE J. Quantum Electron., vol. 31, pp. 2208-2214, 1995.

[9] - "Measurements of the divergence evolution of a copper-vapor laser output by using a cylindrical imaging technique," Appl. Opt., vol. 32, pp. 2058-2061, 1993

[10] J. J. Chang, "Time-resolved beam-quality characterization of copper-vapor lasers with unstable resonators," Appl. Opt., vol. 33, pp. 2255-2263, 1994.

[11] R. Pini et al., "High efficiency diffraction limited operation of a copper vapor laser," Opt. Commun., vol. 81, pp. 138-142, 1991.
[12] P. Coutance et al., "A copper 'HyBrID' laser of high-beam quality using an axial unstable cavity," IEEE J. Quantum Electron., vol. 31, pp. 1747-1752, 1995.

[13] M. J. Withford and D. J. W. Brown, "A $60 \mathrm{~W}$ high-beam-quality singleoscillator copper vapor laser,” IEEE J. Quantum Electron., vol. 35, pp. 997-1003, 1999.

[14] D. J. W. Brown et al., "High-Power, high-brightness master-oscillator power-amplifier copper laser system based on kinetically enhanced active elements," IEEE J. Quantum Electron, vol. 37, pp. 518-524, 2001.

[15] D. W. Coutts and D. J. W. Brown, "Formation of output in copper vapor lasers," Appl. Opt., vol. 34, pp. 1502-1512, 1995.

[16] G. P. Hogan, Ph.D. dissertation, Oxford Univ., Oxford, U.K..

[17] D. R. Jones, "Influence of the Remnant electron density on the performance of copper hybrid lasers," Opt. Commun., vol. 111, pp. 394-402, 1994.

[18] P. G. Foster et al., "Beam characterization and drilling performance of copper bromide lasers," in Proc. ECLAT 1998, B. L. Mordike et al., Ed., Hannover, Germany, pp. 555-560.

[19] R. J. Carman et al., "Influence of the pre-pulse electron density on the performance of elemental copper vapor lasers," Opt. Commun., vol. 157, pp. 99-104, 1998.

[20] - "Modeling the plasma kinetics in a kinetically enhanced copper vapor laser utilising $\mathrm{HCl}+\mathrm{H}_{2}$ admixtures," IEEE J. Quantum Electron., vol. 36, pp. 438-449, 2000.

[21] N. V. Sabotinov, private communication.

Peter Graham Foster was born in Adelaide, Australia, in 1969. He received the B.S. (Hons.) degree in physics from the University of Adelaide, Adelaide, Australia, in 1992, where he is currenlty completing the Ph.D. degree. His research involves the generation of high-beam-quality emission from $\mathrm{CuBr}$ lasers and their application to micro machining.

He was a Research Scientist at Norseld Laser Systems, Adelaide, South Australia, during 1992-1998, developing medical and industrial laser systems for a variety of applications. In 1998, he was Guest Scientist at the University of Bayreuth, Bayreuth, Germany, working on material processing applications of high-power industrial lasers. He is currently Vice President of Fiberbyte, Adelaide, Australia. His research interests include material processing and integrated photonic communication devices. He is the author of over ten scientific publications and conference contributions and holds two patents.

Peter Eric Davis was born in Adelaide, Australia, in 1943. He received the B.E. degree from SAIT in 1979.

He was a Senior Process Electronic Engineer for General Motors Holden. In 1983, he moved to Quentron Optics, where he became manager of the R\&D group in 1985. In 1988, he formed Norseld Laser Systems, Adelaide, Australia, with the aim of developing copper bromide laser systems. His current interests include medical and industrial applications of copper-bromide laser technology.

Mr. Davis is a member of the Institute of Engineers. 
Donald McCoy was born in Adelaide, Australia, in 1936. He received the B.Sc.(Hons.) degree in physics from the University of Adelaide, Adelaide, South Australia, in 1961 and the Ph.D. degree in solid-state physics in 1966.

$\mathrm{He}$ is currently a Senior Lecturer in physics at the University of Adelaide. His research interests include molecular spectroscopy, archaeometry, and lasers. He is the author of over 30 publications.
Jesper Munch was born in Denmark in 1945. He received the B.S. degree from Massachusetts Institute of Technology, Cambridge, and the M.S. and Ph.D. degrees from the University of Chicago, Chicago, IL.

He holds the Chair of Experimental Physics at Adelaide University, Adelaide, Australia, where he has established a research group concentrating on the physics of lasers, frequency stabilization, nonlinear optics, holography, photonics, and general optics. Prior to moving to Adelaide in 1990, he worked for 16 years at TRW, Redondo Beach, CA, where he was a Senior Scientist working on laser development and nonlinear optics. 